\title{
$\eta$ Car: the optical features at the 2003.5 low-excitation event
}

\author{
A. M. van Genderen ${ }^{1}$, C. Sterken ${ }^{2, \star}$, W. H. Allen ${ }^{3}$, and W. Liller ${ }^{4}$ \\ 1 Leiden Observatory, Postbus 9513, 2300RA Leiden, The Netherlands \\ 2 Vrije Universiteit Brussel, Pleinlaan 2, 1050 Brussels, Belgium \\ 3 Alvine Estate, Vintage Lane, RD 3, Blenheim, New Zealand \\ ${ }^{4}$ Casilla 5022, Reñaca, Viña del Mar, Chile
}

Received 2 October 2003 / Accepted 31 October 2003

\begin{abstract}
U B V$ and $B V R$ photometry of $\eta$ Car during the 2003.5 low-excitation event - the considered periastron passage of a binary - is presented. The light and colour curves show a number of features, which were also seen at previous periastron passages: a light maximum of long duration with a superimposed flare-like event which is temporarily interrupted by an eclipselike dip, and a steep decline in the $U-B$ color index. The $R$ brightness reached a minimum at the time of mid X-ray totality, probably implying that the $\mathrm{H} \alpha$ emission line reached a minimum. The source of the optical flare-like event is probably not the same as the one causing the the X-ray radiation. It is tempting to consider the epoch of the $R$ minimum and the mid- $\mathrm{X}$ ray totality - which roughly coincides with the $U B V$ minimum - as the central moment of the 2003.5 low-excitation event.
\end{abstract}

Key words. technique: photometric - stars: individual: $\eta$ Carinae - variables - supergiants

\section{Introduction}

In a recent paper van Genderen et al. (2003, Paper I) discussed the light peak that occurs at the low-excitation events - the supposed periastron passages (PP) of a binary inside $\eta \mathrm{Car}-$ in particular those of 1987.0, 1992.5 and 1998.0. For the impendent event of 2003.5, a campaign was organised to cover this passage in a more complete way than the previous ones. In the mean time, reports on the spectroscopic-, X-ray radiation, radio- and NIR campaigns revealed that the event happened in the expected time interval as predicted by Damineli et al. (2000).

We report here the photometric $(U B V$ and $B V R)$ results of the 2003.5 PP. All observations will be presented in a data paper (Sterken et al., in preparation).

\section{The observations}

\subsection{The UBV photometry}

The observations were obtained with a private $32-\mathrm{cm} f 15$ Dall Kirkham cassegrain telescope situated in Rapaura, Marlborough, New Zealand (latitude $-41^{\circ}$ ). The telescope is equipped with a photoelectric photometer (EMI 9789QB photomultiplier) and a Johnson $U B V$ filter system. The size of the aperture used is $60^{\prime \prime}$. The photometric system was calibrated before this series of observations using standard E region stars

Send offprint requests to: A. M. van Genderen,

e-mail: genderen@strw.leidenuniv.nl

* Research Director, Belgian Fund for Scientific Research.
(Menzies et al. 1989). As shown in Paper I our $U-B$ values for $\eta$ Car are systematically too blue by $\sim 0$. 25 , partly due to filter transmission differences with respect to the standard $U B V$ system and by the presence of emission lines. The question whether this is still the case awaits a comparison with the results of other observers. The observations were made with respect to two comparison stars $\mathrm{A}=\mathrm{HD} 93010(V=6.339$, $B-V=0.019, U-B=-0.764)$ and $\mathrm{B}=\mathrm{HD} 93502(V=6.221$, $B-V=0.061, U-B=-0.307)$, according to the sequence: sky, comp. A, variable, comp. B, variable, comp. A, sky, etc. The sky measurements were always made at the same location near HD 93010. Each $U B V$ measurement is the average of three 10-s integrations (Allen 1989), and each $V, B-V$ and $U-B$ data point is the average of three or four $U B V$ sets relative to the comparison stars. The resulting average standard deviation $(\sigma)$ amounts to $0.02,0.035$ and 0.055 for $V, B-V$ and $U-B$, respectively. The five data points in the very beginning (Fig. 1) show more scatter because the high voltage was lower than for the remaining measurements. A number of times two data points were obtained in the same night, and some of these measurements are so close that they became indistinguishable in the figures.

\subsection{The BVR photometry}

$B V R$ photometry was carried out with an ST-237A CCD camera (TI TC-237 chip) operating at the prime focus of a $203-\mathrm{mm}$ $f 1.5$ Schmidt camera located in Viña del Mar at latitude $33^{\circ} \mathrm{S}$. Comparison stars were HD $93403(V=7.316, B-V=0.219)$ and HD $93737(V=6.048, B-V=0.274)$. Because of the large 


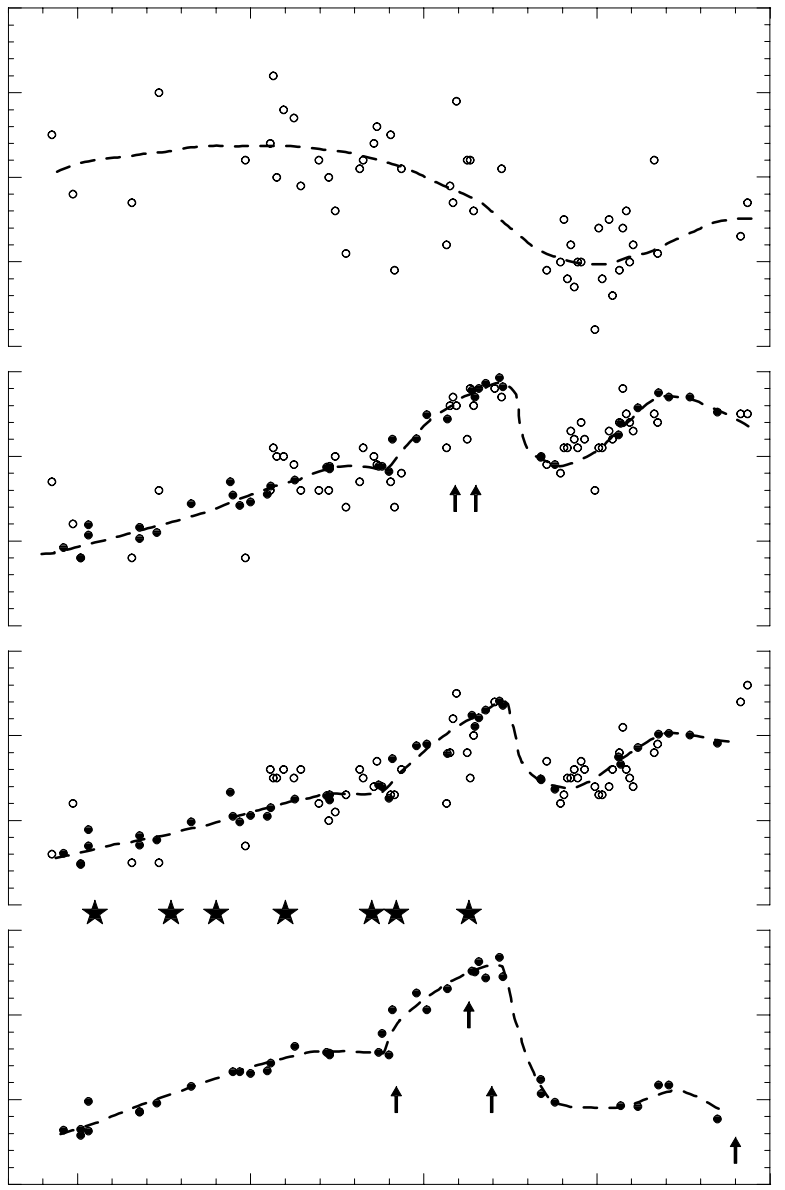

Fig. 1. $U B V(\bullet)$ and $B V R(\circ)$ light curves of $\eta$ Car around the 2003.5 PP. Arrows below the $V$ curve indicate the start of the declines in $L$ and $J$, respectively. Star symbols: dates of X-ray flares. Arrows below the $U$ curve: date X-ray decline, disappearance [NIII] line, start and end of X-ray totality.

field of view $\left(41^{\prime} .3 \times 54.8\right)$, both comparison stars and $\eta$ Car could be imaged in the same frame. Typically, an observation consisted of the sum of 15-20 individual exposures, and magnitudes were derived through aperture photometry. Because the comparison stars did not differ greatly in color from $\eta$ Car, and because both were within a half degree of $\eta$ Car, no extinction corrections were attempted. Repeated observations showed that the standard deviations of the $B, V$ and $R$ measurements were typically $\pm 0.07, \pm 0.03$ and \pm 0.06 magnitudes respectively. The large errors are to be ascribed to the short focal length of the telescope which causes substantial undersampling, even though out of focus stellar images were used. The generally high humidity owing to the proximity of the Pacific Ocean is also believed to contribute to these relatively large uncertainties.

On two occasions (JD 2452813 and JD 2452873 ) a lowdispersion spectrum was observed through the $R$ filter.

\section{Discussion of the light and colour curves}

Figure 1 shows the $R, V, B$ and $U$ light curves for the time interval JD $2452690-2890$. The $V$ and $B$ scales of the 32-cm and
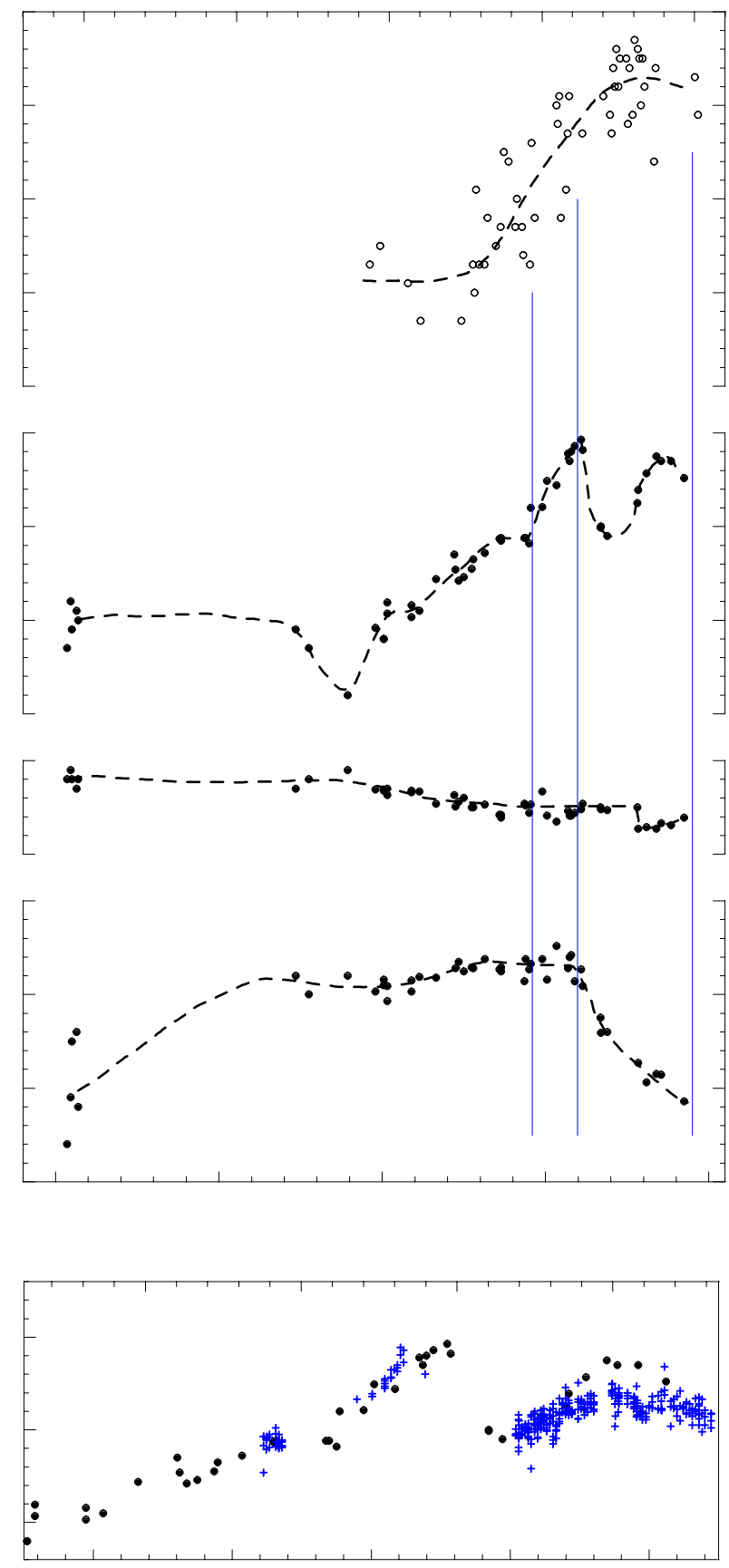

Fig. 2. Top: light and colour curves $(\bullet U B V, \circ B V R)$. Vertical lines: start of X-ray decline, start and end of the X-ray totality. Bottom: $V$ light curve $(\bullet)$ versus the $1998.0 y$ light curve $(+)$ as shown in Fig. 2 of van Genderen et al. (2003). The $y$ zeropoint has been shifted in order to match the $V$ scale ( $y$ diaphragm is $22^{\prime \prime}$ ), the upper $X$-axis has been shifted by $5.5 \mathrm{yr}$.

20 -cm telescopes appeared to differ by 0.09 and 0.07 , respectively, those of the $20-\mathrm{cm}$ telescope being fainter. The magnitudes of the latter were shifted in order to match those of the $32-\mathrm{cm}$ telescope. Arrows and stars indicate a number of epochs related to important features in other wavelenghts (see caption). The $R$ light curve is clearly different from the three other ones: the brightness declines after JD 2452750 until it reaches a 
minimum ( JD 2 452 850). This turns out to be roughly the centre of the X-ray totality (Corcoran 2003). The $R$ brightness is strongly influenced by the decreasing $\mathrm{H} \alpha$ emission line, which contributed approximately $40 \%$ of the total signal of the star at JD 2452813 , see the substantial change of this line between June 1992 and May 1995 as observed by Damineli et al. (1998). Figure 2 shows the $V$ light and colour curves from the $U B V$ data set, and in addition the $V-R$ colour curve. The $R$ brightness decline is evident from the blueing trend of $V-R$.

The low-excitation events or PPs are often marked by a long-term light maximum probably due to an S Dor-phase (van Genderen et al. 2001), the same appears to be the case for the 2003.5 PP (Figs. 1 and 2). During the brightness rise, the $B-V$ became redder as it should for an S Dor-phase, as the $b-y$ did on its way to the 1998.0 PP (Sterken et al. 1999a,b 2001). In our view, the subsequent blueing, accompanying an astonishing continuation of the brightening after the 1998.0 PP, which has never been seen during previous PPs (Davidson et al. 1999; Sterken et al. 1999a,b; 2001), was due to some sort of unusual stellar process, that overwhelmed the typical S Dor-type trends in light and colours. The $U-B$ should show a reddening also, but Fig. 2 indicates the reverse, presumably by an increase of the Balmer continuum radiation of an extended hot plasma, surpassing the typical S Dor-type reddening, until a maximum negative value is reached during the PP. This behaviour is followed by a sudden steep reddening coinciding the start of the $\mathrm{X}$-ray totality. The same has been witnessed for the equivalent colour indices in other photometric systems during previous PPs (van Genderen at al. 1999). In the course of the $5.5 \mathrm{yr}$ cycle, the $U-B$ (and equivalent colour indices) recovers and reaches again a maximum negative value during the next PP.

Smith et al. (2003) assume that the primary is the main source of the UV radiation, that a shell-ejection occurs during the PPs and that its stellar wind shows a strong latitude dependency. This model is appealing in view of the observed $S$ Dor-phases during the PPs (see above) after which an ejection of a shell sounds plausible. Further, the shell-ejections might explain the sudden drop in $U-B$ and its $5.5 \mathrm{yr}$ periodicity, but one should also expect a significant reddening in $B-V$, which is not the case. How to explain with this model the "half-acycle-out-of-phase" phenomenon in the near-UV discussed by van Genderen et al. $(1999,2001)$ is another problem.

Like during the other PPs discussed in Paper I, a 0 . 1 flarelike event occurred. It started at the X-ray decline, suggesting a different origin for both sources (see also point 5 . below). This is the best recorded flare-like event to date. A few particulars should be discussed.

1. $B-V$ and $U-B$ hardly changed during the event, while the one of 1998.0 recorded in the Geneva system, showed a 1.5 times larger rise in the $[U]$ passband (by 0.08 ) than in the six other passbands $(\sim 0$. 05$)$ of that system (van Genderen et al. 1999). The explanation is probably that this passband contains, relative to the broader $U$ band of the $U B V$ system, much more radiation from the Balmer continuum than radiation longward of the Balmer limit. We suggested that a hot spot could have been created in an interacting binary during the close encounter (van Genderen et al. 2001; Paper I). The same suggestion has been made by Feast et al. (2001) on account of their NIR results.

2. $U-B$ shows a sudden decline ( JD 2452815$)$ more or less simultaneously with the light maxima of the flare-like event (Fig. 2). The total decline in $U(0.17)$ is about oneand-one-half times deeper than in $B$ and $V$. The decline in the Geneva $[U]$ during the 1998.0 PP was even two times deeper $(0.12)$ than in the six other passbands $(\sim 0.06)$. For $u$ this amounted to a factor of two as well. Before the decline in $U-B$, the trend to become bluer ceased since about two months. This suggests that the obscuration of the hypothetical hot spot has begun by relatively transparent plasma that is able to absorb Balmer continuum radiation, e.g. by part of a stellar atmosphere, or alternatively by the edge of the interface of the colliding winds. This plasma is obviously not yet dense enough to stop the brightness rise in $V, B$ and even $U$ until the eclipse-like dip.

3. The brightness decline in $V, B$ and $U$ started about two weeks after the NIR decline (Whitelock et al. 2003) and about $10 \mathrm{~d}$ after the disappearance of the N[III] highexcitation line (Damineli et al. 2003), almost together with the $U-B$ decline (see point 2.) and the start of the X-ray totality ( JD 245 2819.66, Corcoran 2003, private communication). The depth of the eclipse-like event is $0^{\mathrm{m}} 1$ in $V$ and $B$, and $0^{\mathrm{m}} .17$ in $U$.

4. After the minimum ( JD 2452840 ), the brightness recovers (mostly in $V$ ), representing the egress without reaching the brightness attained just before the eclipse. We presume that this recovery still represents the flare-like event, but weaker. Hereafter, it seems to fade away.

5 . The X-ray flares indicated by the star symbols below the $B$ curve in Fig. 1 (Corcoran 2003) have no counterparts in the optical, suggesting that the X-ray source is not the source for the optical flare-like event (see discussion just above point 1.).

\section{Conclusions}

Paper I had identified important commonalities of earlier lowexcitiation events which were interpretable as periastron passages in a binary system. These signatures found now confirmation in the new data presented here as the appearance of a flare-like event during the 2003.5 PP. Two other anticipations from that paper came true as well:

a) The flare-like event appears on top of a long-duration light maximum, the first due to the creation of a hot spot in an interacting binary. An S Dor phase, tuned by tidal forces of an approaching companion, is presumably the main underlying cause of the long light maximum.

b) $U-B$ reached the largest negative value just before the start of the X-ray totality, presumably due to the increasing Balmer continuum radiation by hot plasma. This extremum in $U-B$ is followed by a sudden and fast reddening. Since the recovery of $U-B$ takes the complete $5.5 \mathrm{yr}$ cycle, the collapse is not only due to the short lasting eclipse by dense plasma or the like, but presumably also by a large-scale change of extended plasma structures. 
From the somewhat convex shape of the three flare-like events in $V B U$ (see also point 2. in Sect. 3) we get the impression that the obscuration (culminating in the eclipse-like dip) started already during the rise to maximum, thus more or less simultaneously with the X-ray decline. This suggests that the same plasma complexes could have been responsible for both obscuration phenomena, but that does not imply that the source of the optical flare and the X-ray radiation are the same (see below).

According to the minimum in $R$, we presume that the $\mathrm{H} \alpha$ emission reached a minimum roughly at mid X-ray totality (just like during the 1998.0 PP). It thus seems that the screening of high-energy photons is the main cause of the decrease of the $\mathrm{H} \alpha$ emission from the outer layers and the wind of the primary (see also Damineli et al. 1998). It is tempting to consider the epoch of the $R$ minimum ( $\sim \mathrm{JD} 2452850$ ) and the mid-X-ray totality, which roughly coincides with the $U B V$ minimum, as the central moment of the PP. We also point out that the time interval between the $y$ maximum observed at 1998.0 and the $V$ maximum reported here, amounts to 2042 days (see Fig. 2), this is longer by about 3 weeks than the canonical period and illustrates that the creation of the flare-like event depends on varying conditions near the central star.

We agree with studies based on observations at other wavelengths, that a binary revolution as a major cause of the above features is hardly deniable, despite a number of difficulties raised by some spectroscopic and theoretical considerations. Further, we argue that single unstable massive stars, like $\mathrm{S}$ Dor variables (LBVs), with periodic phenomena do not exist. At most their behaviour is quasi-periodic or cyclic (van Genderen 2001; Sterken 2003).

The light curve features of the flare-like event, such as the epochs of the extrema, in the NIR and the optical could well be different depending on the physics of the source (Fig. 1). However, relative light-time effects between the NIR and the optical light should not be excluded. After all, the NIR radiation is more concentrated to the centre. Light-time effects leading to smearing of optical light variations are probably small, but certainly not nil, as explained in our earlier papers.

The optical flare-like event started when the X-ray radiation declined and the X-ray flares (epochs marked in Fig. 1) have no counterparts in the optical, thus, it is not likely that the optical flare-like event and the X-ray radiation come from the same area. The X-ray source is thought to be due to shocked gas forming an interface between two colliding winds (e.g. Corcoran et al. 2001a,b; Stevens \& Pittard 1999). It is conceivable that the hot spot is created in the interbinary medium by an increased mass flow from the primary, but by viewing the orbit under an angle of $49^{\circ}$ (Davidson et al. 2001) during the PP, it might appear to lie close to the X-ray source. Hot spot and $\mathrm{X}$-ray source may then be obscured more or less by the same plasma clouds and more or less at similar epochs.
The interpretations of the discussed details of the multicolor light curves remain partially speculative which is to some extend inherent to the complexity of the $\eta$ Car system. Only the eventual combination of the wealth of data obtained by different groups might shed more light on the nature of the event itself, and the data presented here will be a significant piece in this puzzle. One should, however, take care when comparing and combining photometric data from different sources: $\eta \mathrm{Car}$ no doubt is the single most difficult star to unveil its true standardised magnitude and colours, and before uniting light- and colour curves, one should be aware that systematic differences exceeding 0 m. 05 will occur in the visual magnitudes and colour indices, even more so in the bands that inlude $\mathrm{H} \alpha$. For a profound discussion of these problems, we refer to Sterken (2000) and Sterken et al. (1999a, 2001).

Acknowledgements. This work has been supported by "IUAP P5/36" of the Belgian Federal Office for Scientific, Technical and Cultural Affairs, the Belgian Fund for Scientific Research (FWO) and the Flemish Ministry for Foreign Policy, European Affairs, Science and Technology, under contract BIL 01/2.

\section{References}

Allen, W. H. 1989, Southern Stars, 32, 159

Corcoran, M. F. 2003, Website modified 16 September 2003

Corcoran, M. F., Ishibashi, K., et al. 2001a, ApJ, 547, 1034

Corcoran, M. F., Swank, J. H., et al. 2001b, ApJ, 562,1031

Damineli, A., Kaufer, A., Wolf, B., et al. 2000, ApJ, 528, L101

Damineli, A., Leister, N., Levenhagen, R. 2003, IAUC, 8162

Damineli, A., Stahl, O., Kaufer A., et al. 1998, A\&AS, 133, 299

Davidson, K., Gull, T. R., et al. 1999, AJ, 118, 1777

Davidson, K., Smith, N., Gull, T. R., et al. 2001, AJ, 121, 1569

Feast, M. W., Whitelock, P. A., et al. 2001, MNRAS, 322, 741

van Genderen, A. M. 2001, A\&A, 366, 508

van Genderen, A. M., de Groot, M., Sterken, C. 2001, ASP Conf. Ser., 233, 55

van Genderen, A. M., Sterken, C., Allen, W. H. 2003, A\&A, 405, 1057 (Paper I)

van Genderen, A. M., Sterken, C., de Groot, M., Burki, G. 1999, A\&A, 343,847

Menzies, J. W., Cousins, A. W. J., Banfield, R. M., Laing, J. D. 1989, S. A. Astron. Obs. Circ., 32, 1

Smith, N., Davidson, K., Gull, T. R. , et al. 2003, ApJ, 586, 432

Sterken, C. 2000, IBVS, 5000

Sterken, C. 2003, ASP Conf. Ser., 292, 437

Sterken, C., Freyhammer, L. M., Arentoft, T., van Genderen, A. M. 1999a, A\&A, 346, L33

Sterken, C., van Genderen, A. M., de Groot, M., Burki, G. 1999b, ASP Conf. Ser., 179, 200

Sterken, C., Freyhammer, L. M., Arentoft, T., et al. 2001, ASP Conf. Ser., 233, 71

Stevens, I. R., Pittard, J. M. 1999, ASP Conf. Ser., 179, 295

Whitelock, P. A., Marang, F., Crause, L. 2003, IAUC, 8160 